\title{
LA IMPLEMENTACIÓN DE LOS PRINCIPIOS RECTORES SOBRE EMPRESAS Y DERECHOS HUMANOS EN HONDURAS. UN ANÁLISIS DE LOS ELEMENTOS CLAVE PARA SU PUESTA EN MARCHA
}

\author{
THE IMPLEMENTATION OF THE GUIDING PRINCIPLES ON BUSINESS \\ AND HUMAN RIGHTS IN HONDURAS. \\ AN ANALYSIS OF THE KEY ELEMENTS FOR ITS IMPLEMENTATION
}

\author{
Alejandra Paola López Vásquez ${ }^{1}$ \\ DOI: https://doi.org/10.5377//rd.v40i1.8914
}

\section{RESUMEN}

El presente artículo presenta los resultados alcanzados por el proyecto de investigación científica, no publicado, titulado "La implementación de los Principios Rectores sobre Empresas y Derechos Humanos en Honduras. Un análisis de los elementos clave para su puesta en marcha". Dicha investigación tiene como objetivo principal examinar la importancia y utilidad de los Principios Rectores sobre Empresas y DDHH, y las iniciativas desarrolladas en el contexto internacional para su implementación, e identificar los elementos que de acuerdo a la presente investigación deberán ser tomados en cuenta, al construir una propuesta que permita facilitar la implementación de los Principios Rectores sobre Empresas y Derechos Humanos de la Organización de las Naciones Unidas, que regulan la relación entre la actividad de las empresas trasnacionales y los derechos humanos.

PALABRAS CLAVE: Principios Rectores sobre Empresas y DDHH, Derechos Humanos.

\begin{abstract}
This article presents the results achieved by the scientific research project, unpublished, titled "The implementation of the Guiding Principles on Business and Human Rights in Honduras. An analysis of the key elements for its implementation". Its main objective is to examine the importance and usefulness of the Guiding Principles on Business and Human Rights, and the initiatives developed in the international context for its implementation, and identify the elements that according to the present investigation should be taken into account, when constructing a proposal that facilitates the implementation of the Guiding Principles on Business and Human Rights of the United Nations Organization, which regulate the relationship between the activity of transnational corporations and human rights.
\end{abstract}

KEYWORDS: Guiding Principles on Business and Human Rights, Human Rights

Fecha de recepción: 31 de agosto del 2019

Fecha de aprobación: 31 de septiembre del 2019

\footnotetext{
${ }^{1}$ Profesora Titular de la Universidad Nacional Autónoma de Honduras, Magister en Derecho Empresarial, correo:alejandra.lopez@unah.edu.hn
} 


\section{INTRODUCCIÓN}

El presente artículo científico sistematiza y analiza la investigación no publicada, realizada durante los años 2017 y 2018 acerca de la aplicabilidad de los principios rectores en Honduras, titulada "La implementación de los Principios Rectores sobre Empresas y Derechos Humanos en Honduras. Un análisis de los elementos clave para su puesta en marcha." Tiene como objetivos: Profundizar acerca de la importancia de la implementación de los Principios Rectores sobre Empresas y DDHH, determinar los elementos clave que permiten, tanto a las Empresas como a los Estados la aplicación de los tres pilares propuestos por los Principios Rectores, para integrarlos a la construcción de la propuesta; identificar los retos, desafíos y oportunidades que enfrenta el Estado de Honduras para la implementación de los Principios Rectores sobre Empresas y Derechos Humanos.

\section{METODOLOGÍA UTILIZADA}

Los objetivos propuestos fueron alcanzados a lo largo de la investigación por medio de un estudio Jurídico-Exploratorio, mediante el estudio, análisis e interpretación de los documentos internacionales desarrollados por la Organización de las Naciones Unidas sobre la implementación de los Principios Rectores sobre Empresas y Derechos Humanos, la consulta de artículos científicos y otra bibliografía consultada contentiva de las experiencias de otros Estados en la construcción de sus Planes Nacionales de Acción como medio para la ejecución y aplicación de los Principios Rectores en sus territorios.

A continuación, nos centraremos en elementos más importantes que arroja dicha investigación.

\subsection{El surgimiento de los Principios Rectores sobre Empresas y Derechos Humanos.}

Estos principios surgen como resultado del nombramiento de John Ruggie como Representante Especial sobre Empresas y Derechos Humanos por parte del Secretario General de la Organización de Naciones Unidas (en adelante ONU) en el año
2005. Ruggie, en el año 2008 elaboró un informe, que sirvió de base para la formulación de la Política Marco para los Derechos Humanos y las Empresas Transnacionales y otras Empresas, conocida también como "Proteger, Respetar, Remediar", la que se fundamentaba en "el deber del Estado de proteger contra los abusos a Derechos Humanos por parte de las Empresas. La responsabilidad empresarial de respetar Derechos Humanos, y el acceso a la remediación ya sea mediante mecanismos judiciales y no-judiciales para las víctimas por violación a Derechos Humanos.” (ONU, 2012, p.1).

Con la aprobación del marco, en su cuarto informe Ruggie plantea que para lograr la "operatividad del marco" es necesaria la creación de unas normas que lo complementen. Surgiendo de esta manera, los Principios Rectores sobre Empresas y Derechos Humanos en el año 2011 mediante la aprobación de una parte del Consejo de DDHH de Naciones Unidas, siendo un avance importante en la medida en que esta guía de implementación ayuda a los Estados y empresas a avanzar en el cumplimiento de sus responsabilidades en materia de DDHH.

Los Principios Rectores no han estado exentos de críticas. Algunos autores como Esteve Moltó, (2011) señalan que no crea nuevas obligaciones en materia de DDHH lo que impide exigir a las empresas su observancia, y asumir un verdadero compromiso de respeto hacia los DDHH.

Al respecto, Ruggie señala que: "los Principios Rectores no pondrán fin a todos los problemas que rodean a los derechos humanos, pero su adopción marca el fin del comienzo.” (ONU, 2012, p.3)

2.2. Los principios rectores sobre empresas y DDHH y los tres pilares: proteger, respetar y remediar. Implicaciones para los Estados y las empresas

En este sentido es importante señalar tal como lo menciona Esparza (2014) "los Principios no buscan crear nuevas obligaciones, ya que nos remiten a obligaciones contenidas en otros instrumentos en 
materia de $\mathrm{DDHH}$, como los que se encuentran en los sistemas operacionales para la protección de los DDHH: el sistema universal, referente a Naciones Unidas y el regional existente en los continentes africano, europeo y americano. A este último le corresponde la Convención Americana de DDHH, que recoge un catálogo de DDHH, los que concuerdan con los protegidos por otros instrumentos internacionales, tal y como lo son los Principios Rectores. (p.4) Esto tiene como finalidad motivar a los Estados a una adopción progresiva de dichos estándares dentro de su normativa relacionada con la temática.

A continuación, veremos algunas de las implicaciones más significativas que demanda la aplicación de los Principios por parte de los Estados.

\subsubsection{Pilar: el deber del Estado de proteger los derechos humanos.}

Este pilar de acuerdo con la ONU (2011a) versa sobre la obligación de que los Estados deben proteger contra las violaciones de los derechos humanos cometidas en su territorio y/o jurisdicción por terceros incluidas las empresas. A tal efecto deben adoptar las medidas apropiadas para prevenir, investigar, castigar y reparar esos abusos mediante políticas adecuadas, actividades de reglamentación y sometimiento a la justicia. Para ello es necesario que el Estado y las instituciones que lo integran cuenten con un sistema normativo interno orientado a prevenir los abusos por parte de las empresas en su territorio. Al respecto Ibáñez, y Ordoñez (2014) dicen: "El papel del Estado en este tema no se agota al otorgar las licencias y permisos para que la empresa entre en funcionamiento...El Estado deberá dejar claro que las actuaciones empresariales son monitoreadas y que ante cualquier desviación habrá una sanción o implicación negativa”. (p.239)

De lo anterior podemos concluir que dicho monitoreo deberá reunir dos requisitos muy importantes: abarcar además de las instituciones estales a las organizaciones de sociedad civil para empoderar a la población en general y en segundo lugar el Estado debe tener una presencia constante, permanente en el acompañamiento a las empresas y a la comunidad, brindando asesoría que garantice el respeto a los DDHH, previniendo que se cometan violaciones dentro de su territorio.

\subsubsection{Pilar: La responsabilidad de las empresas de respetar los derechos humanos}

Este pilar establece a las empresas la obligación de enmarcar sus actividades respetando los DDHH. Ello en virtud de la incidencia que han tenido sus actividades en la comisión de violaciones a los DDHH. Al respecto es importante tener en cuenta que, de acuerdo con Cortés (2016) "si bien los Principios Rectores no imponen obligaciones vinculantes a las empresas, ello no implica que ellas no mantengan deberes frente al Estado y deban responder por potenciales violaciones de DDHH. Debemos recordar que la responsabilidad de respetar los DDHH debe ser aplicada por todos los individuos (incluidas las empresas), más allá de lo que determinan las normas nacionales. De tal modo, la responsabilidad existe con independencia de la capacidad y/o voluntad de los Estados de cumplir con sus propias obligaciones con respecto a los DDHH."

Al respecto los principios rectores establecen para las empresas los lineamientos siguientes:

a). Un compromiso político de asumir su responsabilidad de respetar los derechos humanos;

b). Un proceso de diligencia debida en materia de derechos humanos para identificar, prevenir, mitigar y rendir cuentas de cómo abordan su impacto sobre los derechos humanos;

c). Unos procesos que permitan reparar todas las consecuencias negativas sobre los derechos humanos que hayan provocado o contribuido a provocar.

A continuación, explicaremos en qué consisten cada uno de ellos. Siguiendo a Ibáñez, Pilar y Ordoñez, Victoria, (2014) es importante para las empresas expresar públicamente la intención de respetar los derechos humanos. Este compromiso se extiende al establecimiento de sus relaciones con otras empresas que hayan participado en violaciones a los DDHH. Las empresas para cumplir con la debida diligencia 
deberán, además de evitar cualquier violación a los DDHH en el desarrollo de sus actividades empresariales, identificar los impactos generados a través de sus relaciones comerciales. Cortés (2016) al respecto resalta la importancia de los terceros que participan en la cadena de valor de la empresa o que son socios estratégicos. "Son dos los conceptos clave en este aspecto: la esfera de la influencia y la complicidad."

Para el cumplimiento del inciso b) es importante definir en qué consiste la debida diligencia, ya que tiene un papel crucial en las actividades de la empresa. La ONU (2012) en "La responsabilidad de las empresas de respetar los derechos humanos. Guía para la interpretación”, incluye dos definiciones. La primera definición enmarca la debida diligencia dentro de un contexto general:

"Se ha definido la diligencia debida como "la medida de prudencia, actividad o asiduidad que cabe razonablemente esperar, y con la que normalmente actúa, una [persona] prudente y razonable en unas circunstancias determinadas; no se mide por una norma absoluta, sino dependiendo de los hechos relativos del caso en cuestión".

La segunda definición se enmarca en el contexto de los Principios Rectores:

"La diligencia debida en materia de derechos humanos constituye un proceso continuo de gestión que una empresa prudente y razonable debe llevar a cabo, a la luz de sus circunstancias (como el sector en el que opera, el contexto en que realiza su actividad, su tamaño y otros factores) para hacer frente a su responsabilidad de respetar los derechos humanos."

De la definición anterior y siguiendo a Ibáñez, Pilar y Ordoñez, Victoria (2014) es importante que las empresas se comprometan a

- Prevenir y mitigar potenciales riesgos a los derechos humanos causados directamente por los proyectos y operaciones a desarrollar.
- Tener una política de manejo y prevención de riesgos potenciales a los derechos humanos durante su actividad empresarial.

De lo anterior se desprende que las empresas para desarrollar el proceso de debida de diligencia deben establecer un plan de acción para enmarcar sus actividades en el respeto de los DDHH, que contemple las posibles contingencias que puedan ocurrir durante el desarrollo del proyecto, y la manera de mitigar sus efectos.

Para el cumplimiento del inciso c) es necesario que las empresas instauren un mecanismo de recepción de quejas, o como lo denominan los principios rectores los mecanismos de reclamación de nivel operacional a los que puedan acudir directamente las personas y comunidades que hayan sufrido las consecuencias negativas de las actividades de una empresa.

Estos mecanismos de reclamación a nivel operacional desempeñaran dos funciones muy importantes al respecto: En primer lugar, contribuir a determinar las consecuencias negativas sobre los derechos humanos como parte de la obligación de una empresa de proceder con la debida diligencia en materia de derechos humanos. Concretamente, ofrecer un cauce para que las personas directamente afectadas por las operaciones de la empresa pongan en conocimiento sobre las actividades que consideren que les estén ocasionando o que van a sufrir consecuencias negativas, con el fin de que las empresas, analizando las tendencias y las pautas de las denuncias, puedan identificar problemas sistémicos y adaptar sus prácticas sin ocasionar daños. En segundo lugar, estos mecanismos permiten que la empresa se ocupe de los daños detectados y repare las consecuencias negativas, de forma temprana y directa, a fin de evitar daños mayores y prevenir el incremento de reclamaciones.

Del análisis de los elementos anteriores podemos concluir que es necesario para que las empresas cumplan con la debida diligencia del respeto a los DDHH en su actividad empresarial deberán construir un plan de acción que prevea riesgos provocados por el entorno de la comunidad donde llevara a cabo su 
proyecto, evitar constituir relaciones comerciales con empresas o terceros que estén involucrados en violaciones a DDHH (socios comerciales o terceros que participan en la cadena de valor de la empresa) y establecer un mecanismo de reclamación de nivel operacional al que puedan acudir directamente las personas y comunidades que se consideren víctimas de una violación a sus DDHH ocurrida como resultado de las actividades de la empresa.

\subsubsection{Pilar: Acceso a las vías de reparación} de las víctimas de abusos relacionados con las empresas

De acuerdo con este pilar los Estados tienen deben instaurar vías de reparación accesibles, para que las víctimas de abusos puedan acceder sin dificultades. Los Estados deben tomar medidas apropiadas para garantizar, por las vías judiciales, administrativas, legislativas o de otro tipo que correspondan, que cuando se produzcan ese tipo de abusos en su territorio y/o jurisdicción los afectados puedan acceder a mecanismos de reparación eficaces. (ONU, 2011a).

En este sentido la reparación puede consistir en:

“...disculpas, restitución, rehabilitación, compensaciones económicas o no económicas $\mathrm{y}$ sanciones punitivas (ya sean penales $\mathrm{o}$ administrativas, por ejemplo, multas), así como medidas de prevención de nuevos daños como, por ejemplo, los requerimientos o las garantías de no repetición. Estos procedimientos de reparación deben ser imparciales y estar protegidos contra toda forma de corrupción o intento político o de otro tipo de influir en su resultado.” (ONU, 2011a)

Los procedimientos de reparación deberán ser presentados ante mecanismos de reparación. Estos mecanismos de reparación de acuerdo con Isea Silva (2011) a los cuales podrán acceder los afectados se dividen en tres tipos:

- Mecanismos judiciales estatales: los Estados deben tomar las medidas necesarias para asegurar la efectividad de los mecanismos judiciales nacionales, lo que significa reducir al máximo las barreras legales (por ejemplo, leyes que eximan a ciertas empresas de rendir cuentas por sus impactos en los derechos humanos); las barreras prácticas (como los altos costes de presentar denuncias); y las de otros tipos (por ejemplo, la desigualdad en el acceso a la información), con el fin de que las partes afectadas tengan un acceso efectivo a los mecanismos de reparación.

- Mecanismos extrajudiciales de reclamación del Estado: los Principios Rectores también urgen a los Estados a facilitar mecanismos extrajudiciales de reclamación que sean eficaces y adecuados (por ejemplo, mecanismos administrativos $\mathrm{y}$ legislativos). Lo importante aquí es que las partes involucradas conozcan, confíen y sean capaces de utilizar dichos mecanismos; en este sentido, los órganos nacionales de derechos humanos tienen un rol fundamental.

- Mecanismos de reclamación no estatales: los Principios Rectores resaltan la importancia de que los Estados amplíen los mecanismos no estatales de protección de los derechos humanos frente a las consecuencias negativas provocadas por las empresas. Los mecanismos administrados por las propias empresas (a menudo en colaboración con las partes interesadas), por un grupo multilateral, o por un organismo regional o internacional, pueden ofrecer importantes ventajas como 'la rapidez de acceso y reparación, unos costos reducidos y/o alcance trasnacional' (ONU, 2011b, pág. 28).

2.3. Análisis de Derecho Comparado. Iniciativa de la Comisión Europea en la Estrategia sobre Responsabilidad Social Empresarial. La experiencia de otros Estados. Reino Unido y Colombia

Actualmente luego de la aprobación de los Principios Rectores, varios Estados han elaborado su Plan de Acción Nacional (En adelante PNA): Reino Unido, Países Bajos, Dinamarca, Finlandia, Lituania, Noruega y Suecia, Francia, Bélgica, Grecia, Italia, Irlanda, Eslovenia, Portugal, España, Republica Checa, Republica de Eslovenia, Luxemburgo, Chile, Suiza y Estados Unidos y Kenia. (ACNUDH, 2019) 
Lo que pone de manifiesto que hay un compromiso por parte de los Estados de agregar en sus agendas mecanismos de cumplimiento que garanticen el respeto de los Principios Rectores. A continuación profundizaremos en la iniciativa de la Comisión Europea que le dio impulso a nivel internacional al proceso de implementación de los Principios Rectores.

\subsection{La iniciativa de la Comisión Europea en la Estrategia sobre Responsabilidad Social Empresarial}

La Unión Europea elaboró la Estrategia renovada de la UE para 2011-2014 sobre la responsabilidad social de las empresas, teniendo como objetivo dar cumplimiento al "Tratado de la Unión Europea de alcanzar un desarrollo sostenible y una economía social de mercado altamente competitiva. Asimismo, contribuye a lograr los objetivos de la Estrategia Europa 2020 para un crecimiento inteligente, sostenible e integrador, incluido el de una tasa de empleo del 75 \%.” (Unión Europea, 2011). Para ello invitó a los Estados miembros a elaborar sus PNAs para la implementación de los Principios Rectores. Como resultado de esta iniciativa diferentes estados miembros emprendieron el proceso de elaboración de sus planes.

En este punto es importante definir en que consiste un PNA. Lucena Cid (2017) citando a De Felice y Graf, ofrecen ocho criterios para elaborar los PNA sobre empresa y derechos humanos: (i) los PNAs deberían estar basados en estudios de referencia sobre la materia y el análisis de las lagunas entre los compromisos y una efectiva protección de los derechos humanos; (ii) los PNA s deberían incluir a todas la agencias estatales; (iii) el proceso de elaboración de los PNAs y el seguimiento de los mismos deberían garantizar la efectiva participación de todos los agentes implicados (Multi-stakehorder); (iv) los compromisos consignados en los PNAs deberían ser adecuadamente controlados; (v) los PNAs deberían expresar firmemente el compromiso de implementación de los documentos de Naciones Unidas; (vi) los PNAs deberían adaptarse a la estructura y contenidos de los Principios Rectores de Naciones Unidas. (vii) los compromisos recogidos en el PNAs deberían estar bien definidos con fechas claras de acción, con el objeto de poder realizar un seguimiento y control de los mismos; (viii) el octavo criterio establece que los PNAs potencien la capacidad de promover nuevas iniciativas.

El primer Estado en culminar su PNA y ponerlo en práctica fue el Reino Unido, por ello procederemos a analizar su experiencia.

\subsection{Análisis del PNA del Reino Unido}

En septiembre de 2013 atendiendo la invitación de la Unión Europea el Reino Unido lanzó el Plan de Acción Nacional para la implementación de los Principios Rectores sobre Empresas y Derechos Humanos, convirtiéndose en el primer estado en elaborar su PNA con ese propósito. Siendo el resultado del compromiso público asumido por el entonces Primer Ministro David Cameron en noviembre del año 2011. El proceso comenzó en 2012 y comprendió vastas consultas con un sector muy amplio de sociedad civil, incluyendo representantes de ONG y sindicatos, así como una reunión consultiva con grupos empresariales representantes de pequeñas y medianas empresas y otra con empresas transnacionales más grandes. (CAFOD, 2016). Este aspecto ha sido valorado positivamente, ya que ha tenido en cuenta para el proceso de elaboración y construcción del Plan de Acción a muchos sectores de la sociedad.

Mediante su PNA el Gobierno Británico aspira cumplimentar los Principios Rectores a largo plazo. Sin embargo, ha sido objeto de críticas por parte de algunos autores. "En primer lugar, nos enfrentamos a un documento muy general, que alude a propuestas poco aterrizadas y que, a su vez, no asignan compromisos ni medidas concretas de control. Este es un aspecto primordial ya que garantiza la asunción de responsabilidades y obliga a la rendición de cuentas por parte de los diferentes actores estatales... (Cortés, 2016)

ISSN: 2509-5296 (Impreso) 
Gran parte de las acciones contenidas, tienen un cumplimiento de carácter voluntario, sin contar con un cronograma de ejecución que indique las instituciones encargadas de ejecutar las acciones, indicando los períodos de tiempo en los que deberán ser cumplidas, lo que no se adapta a las disposiciones de organismos como la propia ONU, o el Instituto Danés de Derechos Humanos. Lo que dificulta además el seguimiento y monitoreo del PNA, por parte del Gobierno, las empresas, las organizaciones de sociedad civil y demás sectores sociales involucrados. Además, que el documento, no cuenta con indicadores que permitan reflejar el porcentaje de cumplimiento y/o avance anual de las recomendaciones y buenas prácticas contenidas en el mismo, para presentar un informe anual como un mecanismo de rendición de cuentas.

Este es aspecto es de suma importancia a la hora de implementar un PNA de una Política Pública ya que permite determinar el grado de avance en la ejecución de las acciones, permitiendo a los sectores encargados de dar seguimiento sugerir la adopción de medidas que permitan agilizar la implementación y cumplimiento de las acciones en los periodos de tiempo correspondientes.

Tal como lo resaltábamos anteriormente, el PNA de Reino Unido tiene aspectos positivos, los cuales estimamos que otros estados, como el nuestro podrían replicar en la construcción de sus planes de acción:

- En el caso del Gobierno: Brindar un acompañamiento a las empresas para lograr el cumplimiento de los Principios Rectores, implementar medidas como el proceso de certificación de las empresas de seguridad de conformidad a los estándares en materia de derechos humanos, para incentivar a las empresas, promover actividades de proyectos de sensibilización y para abordar los efectos negativos de la actividad empresarial, entre ellas actividades sobre los derechos humanos de grupos como los pueblos indígenas, las mujeres, las minorías nacionales o de grupos étnicos, las minorías religiosas y lingüísticas, los niños, las personas con discapacidad y los trabajadores inmigrantes y sus familias, implementar mecanismos de consulta previa, libre e informada que sean puestos en práctica por las empresas, en las comunidades donde se apresten a desarrollar sus actividades.

- Respecto de las empresas transnacionales exigir que asuman el compromiso de cumplir con todas las leyes aplicables y respetar los derechos humanos reconocidos internacionalmente, dondequiera que operen, adoptar políticas adecuadas de debida diligencia con el fin de identificar, prevenir y mitigar los riesgos sobre los derechos humanos y comprometerse con supervisar y evaluar su implementación, consultar a las personas que puedan verse afectadas en todas las etapas del diseño y la ejecución de los proyectos, de forma tal que se garantice la participación libre e informada, adoptar o participar en mecanismos eficaces de reclamación que sean transparentes, equitativos y predecibles, con la finalidad de permitir la remediación de cualquier impacto adverso en los derechos humanos que causen o con los cuales contribuyan.

\section{- España}

El borrador del el Plan de Empresas y DDHH fue presentado por la oficina de Derechos Humanos del Ministerio de Asuntos Exteriores en el mes de junio del año 2014. Ello con el fin de ser tramitado para su aprobación por el Consejo de Ministros, desde entonces no se ha logrado establecer un consenso entre las instituciones gubernamentales, la sociedad civil y otros interesados. Fue aprobado en julio de 2017. (ACNUDH, 2019). Cuenta con elementos muy valiosos a tener en cuenta para la construcción de un PNA, al respecto Cortés Cabieses (2016 p. 59-61) resalta los siguientes:

1. Está integrado por una serie de acciones, cuya ejecución se encuentra establecida de forma más clara, indicando las instituciones responsables de su cumplimiento.

2. Se busca trabajar con diferentes grupos sociales y recibir retroalimentación a través de la instauración de espacios de dialogo. Por medio de 
la creación de una Comisión de Seguimiento que convocará a reuniones periódicas a los distintos actores involucrados en la ejecución del plan (organizaciones de sociedad civil, sindicatos, empresas, universidades, entre otros), debiendo tener en cuenta las opiniones de los grupos mencionados anteriormente.

3. Llevar a cabo campañas de información y sensibilización adecuadas para cada uno de los grupos de interés a los que van dirigidas.

4. Implantar un dialogo sectorial con el fin de delimitar lo que se espera de las empresas en cuanto al respeto de los DDHH.

5. Plantea la necesidad de una reevaluación y actualización del PNA.

\section{- Colombia}

En Latinoamérica, Colombia es el pionero en haber emprendido diversas iniciativas encaminadas al respeto de los DDHH en el ejercicio de la actividad empresarial, las que viene desarrollando con anterioridad a la aprobación de los Principios Rectores sobre Empresas y DDHH en 2011.

En 2015 la Consejería Presidencial para los Derechos Humanos presentó el Plan Nacional de Acción en Empresas y Derechos Humanos, como resultado de un proceso participativo de consultas con empresas, organizaciones de la sociedad civil y entidades del gobierno nacional, así como con otros gobiernos y organismos internacionales, con el fin de recoger las visiones, intereses y preocupaciones de todos los actores. Ajustando el documento a aspectos como el enfoque basado en derechos humanos, coherencia con otras normas y estándares internacionales, y un enfoque diferencial que consiste en la implementación de acciones afirmativas basadas en el respeto por las identidades como un paso para superar prejuicios, prácticas discriminatorias $\mathrm{y}$ estigmatizaciones que limitan el ejercicio de los derechos.

El Plan está estructurado para dar cumplimiento a las líneas de acción siguientes:
- Coordinación interinstitucional;

- El Estado como actor económico;

- Participación efectiva de la sociedad civil;

- Orientación del Estado para el respeto a los derechos humanos en la actividad empresarial;

- Debida diligencia en derechos humanos;

- Cultura de derechos humanos y construcción de paz en el sector empresarial;

- Debida diligencia en el sector empresarial;

- Respeto a los derechos humanos como ventaja competitiva;

- Responsabilidad social empresarial y respeto a los derechos humanos;

- Fortalecer los mecanismos judiciales y administrativos de acceso a remediación;

- Fortalecer los mecanismos no judiciales de remediación.

Aspectos positivos y necesarios para replicar en el caso de que a futuro Honduras asumiera el compromiso de implementar los Principios Rectores.

\section{PROPUESTA PARA LA IMPLEMENTACIÓN DE LOS PRINCIPIOS RECTORES EN HONDURAS}

\subsection{Retos, desafíos y oportunidades}

El Estado de Honduras ha enfrentado una serie de situaciones que lo enmarcan dentro lo que algunos autores denominan entorno complejo. Alfonso Monroy (2013, p. 366) citando a InternationalAlert (2008), define un entorno complejo como: "la presencia de uno o más de los siguientes factores: a) presencia de actores armados al margen de la ley, b) economías ilegales, c) débil institucionalidad y/o d) frágil gobernabilidad. Aunque no es necesario que estos se presenten simultáneamente, si existe una estrecha relación entre los mismos."

Algunos de estos elementos se encuentran presentes en la situación de Honduras. “...Es un país de ingreso medio-bajo que se enfrenta a desafíos significativos, con más del 60 por ciento de la población viviendo en pobreza. En zonas rurales aproximadamente uno de cada 5 hondureños vive en pobreza extrema (menos 
de US\$1.90 al día). Desde la crisis económica de 20082009, Honduras ha experimentado una recuperación moderada, impulsada por inversiones públicas, exportaciones y altos ingresos por remesas. En 2017 el país creció un 4.8 por ciento y un 3.5 por ciento en 2018, según las últimas estimaciones, y se prevé que el crecimiento para 2019 sea de 3.6 por ciento. A pesar de que las perspectivas económicas son positivas, Honduras enfrenta los niveles más altos de desigualdad económica de Latinoamérica. Otro de sus grandes desafíos es el alto nivel de crimen y violencia. Si bien en los últimos años el número de homicidios ha disminuido, Honduras sigue teniendo una de las tasas más altas en el mundo: 43.6 asesinatos por cada 100.000 habitantes en 2017, según el Observatorio de la Violencia de la Universidad Nacional Autónoma de Honduras. (Banco Mundial, 2019, párr.5.).

Lo anterior sumado a los problemas de gobernabilidad derivados de los cuestionamientos hechos al proceso electoral realizado en noviembre de 2017 , en el que por primera vez un presidente en ejercicio concurría a una reelección presidencial. (Misión de Observación Electoral Unión Europea, 2017)

Es importante resaltar que a pesar de la complejidad del entorno el Estado de Honduras ha emprendido una serie de iniciativas para impulsar el fortalecimiento del respeto a los derechos humanos. Como la creación de la Secretaría de Justicia y Derechos Humanos (decreto 177-2010, de fecha 7 de octubre de 2010), la aprobación el 22 de enero de 2013 de la Política Pública en Derechos Humanos y Plan Nacional de Acción en Derechos Humanos, mediante el Decreto Ejecutivo No. PCM-003-2013 publicado en el Diario Oficial La Gaceta No. 33,073 de fecha 12 de marzo de 2013, la creación de la Ley de Protección de los y las Defensores de Derechos Humanos, periodistas, comunicadores sociales y operadores de justicia, creada mediante Decreto 34-2015, publicado en el Diario Oficial la Gaceta No. 33,730, el establecimiento de la Oficina del Alto Comisionado de las Naciones Unidas para los DDHH y la Misión Internacional de Apoyo contra la Corrupción. Por lo que la adopción de estas iniciativas puede contribuir al fortalecimiento de la materia en nuestro país.
Dentro de la situación de Honduras, hemos identificado como retos a superar: la corrupción y una presencia institucional débil. Los escándalos de corrupción que han salido a la luz pública en los últimos años demandan garantizar la efectividad de los mecanismos estatales de lucha contra la corrupción (Ministerio Público, Poder Judicial, Comisionado Nacional de Derechos Humanos) con el fin de recobrar la confianza de la población en la institucionalidad, evitando el mal manejo de los fondos del Estado por parte de diferentes funcionarios públicos, y castigando a los culpables, en caso de probárseles su responsabilidad, además que la corrupción ha estado presente en las negociaciones de contratos entre el Estado y las empresas no sólo transnacionales, sino además con empresas nacionales.

Sobre el segundo reto el Estado debe fortalecer su presencia institucional en el interior del país, dado que tendrá que fortalecerla para vigilar que la protección de los derechos humanos sea efectiva, y para impulsar acciones tendentes al desarrollo económico y social de dichas zonas, proclives a las violaciones en materia de DDHH.

\subsection{Elementos a tener en cuenta para la construcción de un PNA sobre Empresas y Derechos Humanos en Honduras}

Con base en el análisis desarrollado a lo largo de la presente investigación somos del criterio que los Principios Rectores podrían ser implementados a futuro en Honduras mediante la construcción de un PNA en materia de Empresas y DDHH cuya implementación tendría que desarrollarse a largo plazo. La institución encargada de coordinar e implementar este proceso sería la Secretaría de Estado en el Despacho de Derechos Humanos.

Para ello estimamos que deberán tenerse en cuenta los siguientes criterios que hemos venido analizando anteriormente:

- El Estado deberá de asumir un compromiso público para garantizar el cumplimiento de los Principios Rectores. 
- El PNA deberá adaptarse a las necesidades políticas, económicas, sociales y culturales del entorno del Estado.

- Deberá hacerse un diagnóstico previo en que se analice la normativa en materia de Derecho Mercantil, Derecho Laboral, Derecho Ambiental, que resulta incompatible y /o contradictoria con los Principios Rectores, políticas públicas $\mathrm{y}$ otros instrumentos existentes y los tratados internacionales en materia de DDHH suscritos por Honduras para proceder a su armonización.

- Elaborar un mapeo de los grupos de interés involucrados en la construcción y ejecución del PNA, como, por ejemplo: instituciones gubernamentales, empresas, organizaciones de la sociedad civil, instituciones universitarias (públicas y privadas) representantes de los diferentes colegios profesionales, sindicatos de trabajadores

- El PNA deberá ser el resultado de un proceso de socialización y dialogo con diversos actores de la sociedad hondureña; instituciones gubernamentales, empresas, organizaciones de la sociedad civil, instituciones universitarias (públicas y privadas) representantes de los diferentes colegios profesionales, sindicatos de trabajadores y organismos internacionales (Oficina Alto Comisionado de las Naciones Unidas, Unión Europea). Tal como se construyó en los estados mencionados anteriormente.

- Este dialogo deberá llevar a la construcción de campañas de información y sensibilización dirigidas a los grupos de interés involucrados con el propósito de delimitar de forma clara y precisa que se espera de las empresas con relación al respeto de los derechos humanos en el desarrollo de sus actividades, las obligaciones del Estado y los mecanismos de remediación a los que pueden acceder las victimas en caso de ocurrir violaciones a los DDHH como resultado de dichas actividades.

- El PNA requerirá un estudio de línea base para establecer una medición de los indicadores contemplados para su desarrollo.

- Deberá contener un cronograma de ejecución, detallando las instituciones que se encargarán de dar cumplimiento a las acciones, así como también los periodos de tiempo en los que deberán ejecutarse las acciones correspondientes. Estas instituciones pueden ser las involucradas en la red de puntos focales de las Secretarías de Estado, órganos desconcentrados y descentralizados que se les instruye designar a las instituciones antes mencionadas en el artículo 3 del Decreto PCM-28-2017 que da vida al Sistema de Monitoreo y Seguimiento de las Recomendaciones de Honduras (SIMOREH), el cual surge como resultado de un Programa de Cooperación Técnica para su implementación suscrito entre la República de Honduras y la República de Paraguay. Es importante mencionar que este sistema es una herramienta informática que permite el acceso y monitoreo a través de una aplicación web para la implementación de las recomendaciones formuladas al Estado de Honduras por los mecanismos de protección de los Derechos Humanos, tanto de la Organización de las Naciones Unidas (ONU) y el Sistema Interamericano de Derechos Humanos. (República de Honduras, 2017). Este sistema facilita el seguimiento al cumplimiento de la Política Pública y Plan Nacional de Acción en Derechos Humanos, por lo que a futuro, en caso de implementarse un PNA sobre empresas y DDHH sería una herramienta eficaz para medir los avances en su implementación.

- Contar con mecanismos de rendición de cuentas que faciliten el seguimiento al cumplimiento de las acciones contenidas en el PNA. Este también se podrá desarrollar mediante el SIMOREH, a través de la presentación de informes de carácter anual sobre los avances en la implementación del PNA.

- El establecimiento de una Comité Asesor que vigile, oriente, reformule y dé seguimiento al cumplimiento de las acciones contenidas en el PNA. Este puede ser integrado por organizaciones de sociedad civil, organismos internacionales (Oficina Alto Comisionado de las Naciones Unidas) Unión Europea, instituciones universitarias (públicas y privadas), representantes de los diferentes colegios profesionales y sindicatos de trabajadores. 
- Garantizar el acceso de las víctimas a mecanismos de remediación.

- Deberá contener de acuerdo con Cantú Rivera (2016) ...una combinación inteligente de medidas que incluya enfoques voluntarios y obligatorios, incentivos y sanciones...que ataquen las principales deficiencias de gobernanza, que coadyuvan a la existencia de zonas grises donde ocurren la mayoría de las violaciones empresariales de DDHH.

\section{CONCLUSIONES}

1. Pese a las críticas a los instrumentos que no establecen obligaciones de carácter imperativo (Soft Law) estos instrumentos representan un inicio para la regulación de la actividad empresarial y su relación con el respeto a los DDHH que siempre se ha mantenido convenientemente al margen de todo lo que implique el establecimiento de obligaciones derivadas de su actividad en la materia.

2. Los Principios Rectores tienen un carácter transversal, por lo que guardan una estrecha relación con otras obligaciones derivadas de otros tratados y convenios internacionales, por lo que no crean nuevas obligaciones. Ya que estas se hallan insertas en la legislación vigente del Estado.

3. Los Principios Rectores están siendo implementados por diferentes países alrededor del mundo a través de la construcción de PNAs, los que deben establecer claramente las obligaciones de las empresas en caso de cometer abusos.

4. La creación de un PNA en Honduras para la aplicación y cumplimiento de los Principios Rectores puede fortalecer la articulación de esfuerzos por parte de las instituciones gubernamentales, la academia, organizaciones de sociedad civil, colegios profesionales y sindicatos para el logro de un objetivo común: el respeto de los DDHH, tal como lo evidencia el caso de Colombia. Por lo que sí creemos que puede ser posible su aplicación en nuestro país.
5. Puede contribuir al fortalecimiento de una cultura de respeto a los $\mathrm{DDHH}$, no sólo en el ámbito empresarial sino además en otras áreas que se relacionan: el respeto a los territorios ancestrales de las comunidades indígenas y afrodescendientes a través de mecanismos de consulta previa, el uso racional de los recursos naturales para el impulso de los objetivos de desarrollo sostenible, condiciones de trabajo dignas para los pobladores de los lugares donde se lleven a cabo los proyectos.

\section{RECOMENDACIONES}

1. El Estado de Honduras debe establecer, fortalecer y mantener una cultura de respeto a los DDHH, que le permita recuperar la credibilidad perdida ante la sociedad por actos de corrupción y acontecimientos políticos.

2. Reformar los planes de estudios del sistema educativo en primaria y secundaria, incluyendo la asignatura Derechos Humanos.

3. Es necesario el fortalecimiento, evaluación, readecuación y actualización del Plan Nacional de Acción en Derechos Humanos para que tenga un mayor impacto en su aplicación, ya que cuenta con una serie de acciones muy importantes en el fortalecimiento del tema de derechos humanos y mediante una actualización pueden lograr avances significativos a futuro en el tema de Empresas y DDHH.

4. Aprobar la Ley sobre la Consulta Previa, Libre e Informada a los pueblos indígenas y afrodescendientes, garantizando los mecanismos apropiados para consultar a los pobladores de estas comunidades su aprobación o rechazo a la ejecución de los proyectos empresariales en sus territorios, cuando consideren que son lesivos a sus intereses. La aprobación de esta ley es muy importante para el establecimiento del respeto y debida diligencia con que deben obrar las empresas en el desarrollo de sus proyectos. 
5. Es importante impulsar iniciativas como la implementación del Sistema de Monitoreo y Seguimiento de Recomendaciones (SIMOREH), en virtud de que puede fortalecer no sólo la presentación de informes y el seguimiento de las recomendaciones emitidas al Estado de Honduras por parte de los diferentes órganos de Tratados en materia de DDHH, sino que además puede fortalecer e impulsar la construcción y el fortalecimiento de una cultura de DDHH, incluyendo las Empresas y DDHH.

\section{BIBLIOGRAFÍA}

- Alfonso Monroy, María Paula, (2013). Empresas y Derechos Humanos: una responsabilidad en construcción. Revista de la Facultad de Derecho y Ciencias Políticas, volumen 43, No. 118, Enero-Junio de 2013. Recuperado del sitio web el 27 de marzo de 2017, del sitio web: http://www.scielo.org.co/scielo.php?pid=S0120$38862013000100011 \& \mathrm{script}=\mathrm{sci}$ abstract\&tlng=es

- Alto Comisionado de las Naciones Unidas (ACNUDH), (2019). Planes de Acción Nacionales. Recuperado el 26 de agosto de 2019 del sitio web: https://www.ohchr.org/SP/Issues/ Business/Pages/NationalActionPlans.aspx

- Catholic Agency For Overseas Development (Cafod), ¿Líderes o rezagados?, Recuperado el 24 de enero de 2018, del sitio web: https://cafod.org. uk/content/download/41742/473282/version/1/ file/L\%C3\%ADderes\%20o\%20rezagados $\% 20$ CAFOD $\% 20$ informe $\% 20$ empresas $\% 20 \mathrm{y} \% 20$ derechos\%20humanos.pdf

- Cantú Rivera, Humberto, (2013). Empresas y Derechos Humanos: ¿hacia una regulación jurídica efectiva o el mantenimiento del status quo?, Anuario Mexicano de Derecho Internacional, Volumen XIII, 2013, págs. 315-354, recuperado el 27 de marzo de 2017 del sitio web: http://www. scielo.org.mx/pdf/amdi/v13/v13a7.pdf
- Cantú Rivera, Humberto, (2016). Planes de acción nacional sobre empresas y derechos humanos: sobre la instrumentalización del derecho internacional en el ámbito interno, Recuperado el 20 de enero de 2018 del sitio web: https://www.sciencedirect.com/science/article/ pii/S1870465417300314

- Congreso Nacional de Honduras. (7 de octubre de 2010). Artículo 28 [Sección Tercera: Secretarías de Estado]. Ley General de la Administración Pública [177-2010]. DO: 32,335., recuperado el 2 de febrero de 2018, del sitio web: http://www. poderjudicial.gob.hn/transparencia/regulacion/ diariooficiallagaceta/Documents/Reforma $\% 20$ a $\% 201$ os\%20Art $\%$ C3\%ADculos $\% 2028 \% 20 \mathrm{y} \% 20$ 29\%20del\%20Decreto\%20No\% $2014686 \% 2$ C $\% 20$ contentivo\%20de $\% 201 \mathrm{a} \% 20 \mathrm{Ley} \% 20 \mathrm{General} \% 20$ de \%201a\%20Administraci\%C3\%B3n \%20 P\%C3\%BAblica.pdf

- Congreso Nacional de Honduras (fecha 12 de marzo de 2013). Artículo 1 [Título único]. Política Pública en Derechos Humanos y Plan Nacional de Acción en Derechos Humanos [PCM-0032013]. DO: 33,073. Recuperado el 2 de febrero de 2018 del sitio web: https://www.tsc.gob.hn/leyes/ Aprobar politica publica y plan nacional accion derechos humanos 2013.pdf

- Congreso Nacional de Honduras (fecha 15 de mayo de 2015). Artículo 2 [Título I]. Ley de Protección de los y las Defensores de Derechos Humanos, periodistas, comunicadores sociales y operadores de justicia, [Decreto 34-2015]. DO: 33,73. Recuperado el 2 de febrero de 2018, del sitio web: https://www.tsc.gob.hn/leyes/ Ley Proteccion defensores der humanos periodistas op just.pdf

- Congreso Nacional de Honduras. (7 de agosto de 2017). Artículo 3 [Título único] Establecimiento del Sistema de Monitoreo y Seguimiento de las Recomendaciones de Honduras (SIMOREH), [Decreto PCM-28-2017]. DO: 34,410. Recuperado el 2 de febrero de 2018, del sitio web: http://www.secretariaconsejodeministros.gob. 
hn/sites/decretos/2017/agosto/PCM-28-2017. pdf\#page $=1 \&$ zoom $=$ auto, $-73,792$

- Consejería Presidencial para los Derechos Humanos, (2015). Plan Nacional de Acción en Empresas y Derechos Humanos, Colombia, recuperado el 28 de febrero de 2018 del sitio web: $\quad$ http://www.derechoshumanos.gov.co/ Prensa/2015/Documents/Plan\%20Nacional\%20 de $\% 20$ Accion $\% 20$ DDHH $\% 20$ Empresa.pdf

- Consejería Presidencia para los Derechos Humanos, (2017). Avances y desafíos en la implementación del Plan Nacional de Acción de Colombia, recuperado el 28 de febrero de 2018, del sitio web: https://www.youtube.com/ watch? $=$ tvZnQUJHDkY

- Cortés Cabieses, Micaela, La implementación de los principios rectores sobre las empresas y derechos humanos en el Perú, Tesis, Pontificia Universidad Católica del Perú, Lima, 2016. Recuperada el 30 de junio de 2017, del sitio web: http://tesis.pucp.edu.pe/ repositorio/bitstream/handle/123456789/7110/ CORTES CABIESES MICAELA LA IMPLEMENTACI\%C3\%93N.pdf? sequence $=1$

- Banco Mundial, (4 de abril de 2019). Honduras: Panorama Mundial. Recuperado el 29 de agosto de 2019, del sitio web: https://www.bancomundial. org/es/country/honduras/overview

- Esteve Moltó, José Elías. (2011) Los Principios Rectores sobre las empresas transnacionales y los derechos humanos en el marco de las Naciones Unidas para «proteger, respetar y remediar»: ¿Hacia la responsabilidad de las corporaciones o la complacencia institucional? Recuperado el 5 de julio de 2017 del sitio web: https://www.unav.edu/ publicaciones/revistas/index.php/anuario-espdcho-internacional/article/viewFile/2559/2432

- Esparza, Laura, (2014). Los principios rectores sobre empresas y derechos humanos como instrumento de soft law. Recuperado el 30 de diciembre de 2017 del sitio web: https://www.gob. $\mathrm{mx} / \mathrm{cms} / \mathrm{uploads} /$ attachment/file/47800/Los Principios Rectores y su aplicacion como instrumento de soft law.pdf

- Her Majesty's Government. Buenas prácticas comerciales: Aplicación de los Principios Rectores de la Organización de Naciones Unidas sobre las empresas y los derechos humanos. Recuperado del sitio web: https://www.gov.uk/government/ uploads/system/uploads/attachment data/ file/237053/BHR Action Plan - final online version Spanish LatAm .pdf

- Ibañez, Pilar y Ordoñez, Viviana, (2014). Papel de las empresas y de los Estados en la debida diligencia en derechos humanos. Revista Colombiana de Derecho Internacional, pp.219246. doi:10.11144/Javeriana.IL14-24.pedd

- Instituto Danés para los Derechos Humanos. (s.f.) 10 Criteria for National Action Plans (NAPs) on Business and Human Rights, recuperado el 05 de febrero de 2018, del sitio web: https://businesshumanrights.org/sites/default/ files/documents/10\%20Criteria $\% 20$ for $\% 20$ National\%20Action\%20Plans\%20(NAPs).pdf

- Isea Silva, Ricardo, (2011). Las empresas y los derechos humanos, Cuadernos de la Catedra "La Caixa” de la Responsabilidad social de la empresa y gobierno corporativo, Universidad de Navarra, IESE Bussines School, No. 12, septiembre de 2011. Recuperado el 17 de abril de 2017 del sitio web: www.iese.edu/es/files/cuaderno12 Final tcm5-71129.pdf

- Lucena Cid, Isabel (2017). La Implementación de los Principios Rectores sobre las Empresas y los Derechos Humanos. Implicaciones para los Estados, Universitas Revista de Filosofía Derecho y Política, Universidad Carlos III, Madrid, 2017, No 25 / pp. 69-89, ISSN 1698-7950 / doi: https:// doi.org/10.20318/universitas.2017.3557

- Misión de Observación Electoral de la Unión 
Europea, (2017). Informe Final Elecciones Generales 2017. Recuperado del sitio web: https:// eeas.europa.eu/sites/eeas/files/373041878-moeue-honduras-2017-informe-final.pdf

- Organización de las Naciones Unidas, (2015). Los 10 Principios del Pacto Mundial. Recuperado el 27 de marzo de 2017, del sitio web: http://www. pactomundial.org/2015/02/10-principios-delpacto-mundial/.

- Organización de las Naciones Unidas, (2011a). Principios rectores sobre empresas y derechos humanos. Puesta en práctica del marco de las Naciones Unidas para proteger, respetar y remediar. Nueva York y Ginebra. Recuperada el 29 de diciembre de 2017 del sitio web: http:// www.ohchr.org/Documents/Publications/ GuidingPrinciplesBusinessHR SP.pdf

- Organización de las Naciones Unidas, (2012). La responsabilidad de las empresas de respetar los derechos humanos. Guía para la interpretación. Nueva York y Ginebra. Recuperada el 27 de marzo del sitio web:https://business-humanrights. org/es/node/86208/principios-rectores-sobreempresas-y-derechos-humanos/gu\%C3\%ADa-deinterpretaci\%C3\%B3n-por-las-naciones-unidas

- Organización de Naciones Unidas, (2011b). Informe del representante especial del Secretario General para la cuestión de los derechos humanos y las empresas transnacionales y otras empresas. Principios Rectores sobre las empresas y los derechos humanos: puesta en práctica del marco de las Naciones Unidas para "proteger, respetar y remediar”. Recuperado el 2 de enero de 2018 del sitio web: www2.ohchr.org/SPdocs/Business/A$\underline{\text { HRC-17-31 sp.doc }}$

- Unión Europea, Estrategia renovada de la UE para 2011-2014 sobre la responsabilidad social de las empresas. Recuperado 10 de enero de 2018, del sitio web: http:// eur-lex.europa.eu/legal-content/ES/TXT/ $\underline{\mathrm{PDF} / \text { ?uri=CELEX:52011DC0681\&from }=\mathrm{ES}}$ 